\title{
Statics and dynamics of a nanowire in field emission
}

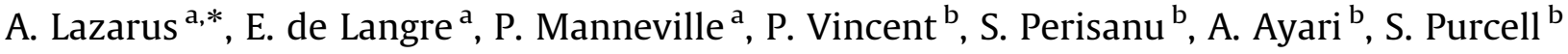 \\ a Laboratoire d'Hydrodynamique, Ecole Polytechnique, 91128 Palaiseau, France \\ ${ }^{\mathrm{b}}$ Laboratoire PMCN, CNRS Universite Lyon 1, 69622 Villeurbanne, France
}

\begin{abstract}
We simulate the nonlinear behaviour of a cantilevered nanowire in field emission to understand and exploit the self-oscillations experimentally observed in this nanoelectromechanical system. Statics and dynamics of this oscillator are predicted with a low-dimensional model consisting of a bi-articulated cantilevered beam flowing electrons and immersed in an electrostatic environment. We set up the qualitative nonlinear governing equations of the system and also highlight the original coupling between the electrostatic field, the nanowire motion and the electric field emission current. A linear stability analysis of the nonlinear static fixed points aims at determining the instability threshold as a function of the applied $D C$ voltage. It is found that instability is mostly due to the competition between the field emission current dependence on the nanowire position and the voltage. As a consequence, the emergence of flutter requires specific external conditions such as an initial angular imperfection, a strong mechanical $Q$ factor or a high electrical resistance. Finally, a direct integration of the nonlinear governing equations confirms the presence of high-frequency self-oscillations, i.e. the possibility of $D C /$ $A C$ conversion in this autonomous electromechanical device.
\end{abstract}

\section{Introduction}

Nanoelectromechanical systems (NEMS) based on mechanical resonances of nanostructures, such as nanotubes or nanowires, are drawing interest from both technical and scientific communities [1]. Their extremely small dimensions make them highly sensitive to external electrostatic perturbations and, due to their outstanding mechanical properties such as strong Young's modulus or high quality factor $Q$ [2] their mechanical response can exceed the quality of electrical signals from purely electronic devices. As well, NEMS oscillators have been proposed for use in ultrasensible mass detection [3] or radio frequency for wireless communication [4].

Among the great variety of these nanocomponents, NEMS based on singly clamped cantilevers in field emission (FE) configuration have recently proven original capabilities [5]. In this FE configuration, a nanotube or nanowire is connected to the cathode and a DC voltage $V$ is applied between the cathode and an anode positioned in the vicinity of the nanostructure (Fig. 1). For a voltage $U_{\text {ref }}$, the electric field at the nanowire apex, enhanced by its tip effect, becomes sufficient to extract electrons by tunnelling effect. This quantum process results in a field emission DC current depending on the applied voltage $V$ [6].

\footnotetext{
* Corresponding author.

E-mail addresses: arnaud.lazarus@polytechnique.edu (A. Lazarus), delangre@ladhyx.polytechnique.fr (E. de Langre).
}

\author{
Keywords: \\ Nanotechnology \\ Electro-mechanical coupling \\ Slender structures dynamics \\ Linear stability
}

One of the originalities when using cantilevered nanostructures as field emitters is that their extreme mechanical sensitivity reveals dependence of the FE current on the position of the emitter. The new NEMS applications in this configuration take advantage of this original coupling between the emitted current and the position of the emitter apex in its environment. One can also mention that this configuration is well adapted for mechanical studies on nanotubes and nanowires, as the resulting patterns of the emitted electrons give a direct projection of the apex motion on a phosphor screen (Fig. 1). This allows investigations of linear and nonlinear behaviour of nanocantilevers [7,8].

A particularly interesting application recently shown by Ayari et al. [9], using highly resistive nanowires, has been the observation of self-oscillations resulting from the electromechanical interactions between the electrical and mechanical properties of the cantilever. They showed that above a critical DC voltage, the nanostructure starts to oscillate resulting in an AC field emission current. The realization of an AC current generator at the nanoscale simply commanded by a DC voltage has interesting potentialities in autonomous nanosystems such as smart dust applications. From a theoretical point of view, this system exemplifies an original coupling that appears at the nanoscale between mechanical behaviour, electrostatic environment and FE properties.

The present paper proposes nonlinear simulations to predict nonlinear statics and dynamics of a nanowire in field emission. Inspired by the fluid-structure interaction formalism for modelling the instability of slender structures in axial flow [10,11], 


\section{Nomenclature}

\section{Kinematics}

D diameter of the nanowire

$L \quad$ length of the nanowire

$q_{1}, q_{2} \quad$ absolute angular displacements

$\theta_{1}, \theta_{2}$ angular displacements around the tilting position

$\psi \quad$ initial angular tilting of the nanowire
Mechanics

$a_{k} \quad$ rotational stiffness ratio of the interconnected springs

$\mu \quad$ viscous damping of the interconnected dampers

$k_{A}, k_{B} \quad$ rotational stiffnesses of the interconnected springs

$m \quad$ nanowire mass per unit length

Q dimensionless mechanical quality factor

$\tau \quad$ dimensionless time scale

$\underline{\varphi}_{1}, \underline{\varphi}_{2} \quad$ shapes of the first and second mechanical mode

$\frac{\varphi}{\omega} 1, \underline{\varphi}_{2}$ reference frequency of the oscillating nanowire

\section{Electrostatics}

$C, c \quad$ electrical capacitance and its dimensionless form

$C_{\text {ref }} \quad$ electrical capacitance when $q_{1}=q_{2}=0$

$\underline{E}, \underline{e} \quad$ electric field and its dimensionless form

$\bar{F} \quad$ electrostatic force applied on the nanowire

$\overline{\mathfrak{M}}_{1 A}, \mathfrak{M}_{2 A}, \mathfrak{M}_{2 B}$ moments of the electrostatic forces about the two articulations $A$ and $B$

$\mathrm{m}_{1 A}, \mathrm{~m}_{2 A}, \mathrm{~m}_{2 B}$ dimensionless forms of the electrostatic moments

$\mathfrak{U}, \mathfrak{u} \quad$ electric potential and its dimensionless form

$S \quad$ boundary of the electrostatic problem

$\beta, b \quad$ field enhancement factor and its dimensionless form

$\beta_{\text {ref }}$ field enhancement factor when $q_{1}=q_{2}=0$

\section{Electricity}

$I_{c} \quad$ capacitor current

$I_{e}, i_{e} \quad$ field emission current and its dimensionless form

$H, G, h, g$ Fowler-Nordheim empirical constants and dimensionless forms

$R \quad$ nanowire electrical resistance

$r \quad$ ratio between electrical and mechanical time scales

$U, u \quad$ voltage at the nanowire tip and its dimensionless

$V, v \quad$ applied $D C$ voltage and its dimensionless form

$U_{\text {ref }} \quad$ field emission voltage reference

$\underline{\varphi}_{3} \quad$ electrical mode shape

we describe a discrete model to understand and also make the most of the physical phenomena involved in this original nanoelectromechanical system. Based on the first investigations by Ayari et al. [9], we extend their model to a rigorous geometrically nonlinear one with richer kinematics. The electrostatic problem, the mechanical behaviour and the field emission influence are successively discussed. This work provides a first

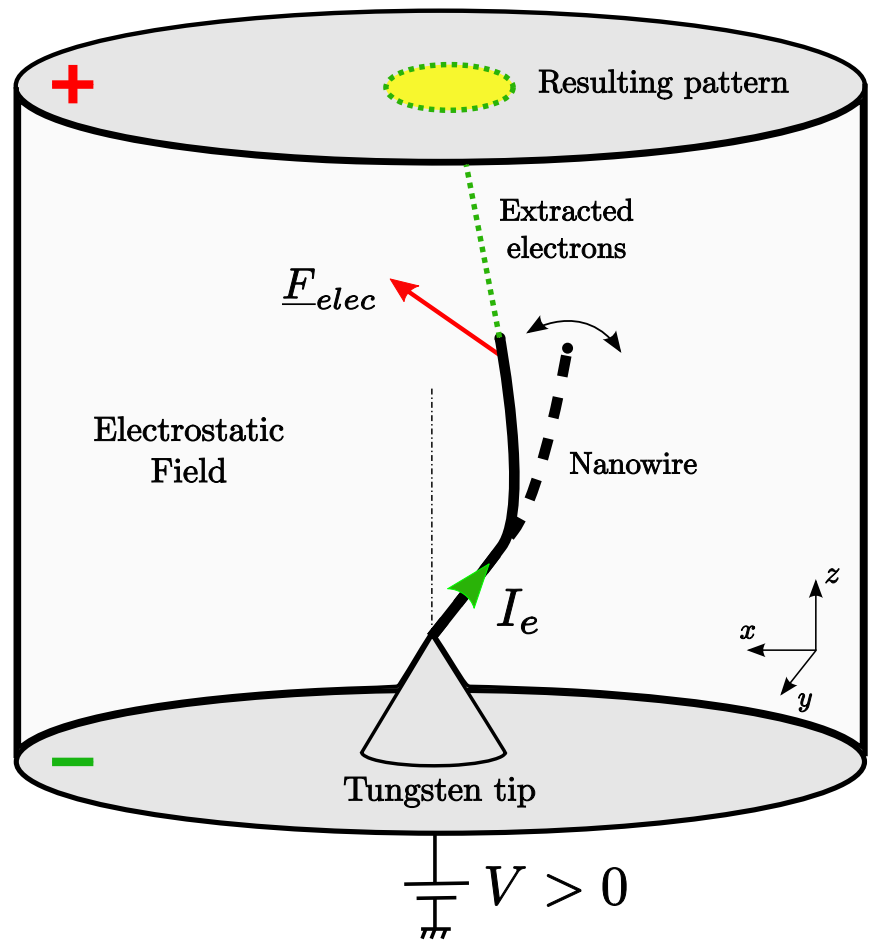

Fig. 1. Experimental settings: nanowire in field emission. qualitative model of a cantilevered nanowire in field emission but can also be extended to the modelling of vibrating nanostructures so far mainly based on linear beam theory [12].

In Section 2, we set up the general governing equations of a biarticulated nanowire in field emission, which is expected to be a suitable low-dimensional model to compute qualitative results. The electrostatic environment of the nanostructure governs the applied electrostatic forces and some essential electrical properties respectively required to set up the coupled mechanical and electrical models. The complete dimensionless nonlinear formulation of the discrete system is given at the end of the section. We then specify, in Section 3, the parameters of the model related to the physical problem for further computations. The chosen kinematics leading to a low-dimensional governing equation, many discrete electromechanical parameters are determined through experimental observations of the continuous system. The remaining unknown quantities are obtained by computing the electrostatic problem. In Section 4, we perform the numerical simulation of a nanowire in field emission. We confirm the possibility of self-oscillations around the system static equilibrium. An explanation of the physical phenomenon responsible for the instability is suggested in Appendix A. A parametric study of the NEMS linear stability allows us determining the electrical and mechanical parameters leading to self-oscillations. Finally, implicit time integration is performed to simulate the limit cycles and the output $A C$ signal of the nanodevice at the instability threshold.

\section{Fully nonlinear model of a nanowire in field emission}

In order to set up the governing equations of the nanowire in field emission, one needs first to sort out the strong connections between the different physical problems contained in the system, i.e. the electrostatic, the mechanical and the electrical ones. In the following, after choosing an appropriate kinematics, we show 
how to compute the electrostatic problem giving us the required variables to study further the dynamics of our nanoemitter. Indeed, once this done, the nanowire motion and its electrical equilibrium are completely defined at any instant $t$ and the dimensionless governing equations of the electromechanical system can then be given.

\subsection{Kinematics}

The diagram in Fig. 2a illustrates the kinematics chosen to model the dynamical behaviour of a cantilevered nanowire with length $L$ and diameter $D$ in the field emission setup presented in Fig. 1. The geometrical configuration of the oscillating nanowire is described at any instant $t$ by two generalized coordinates $q_{1}$ and $q_{2}$ such that

$q_{1}(t)=\theta_{1}(t)+\psi$,

$q_{2}(t)=\theta_{2}(t)+\psi$,

where the constant $\psi$ is the initial angle made by the nanowire with the $z$-axis considered as the symmetrical FE configuration. Indeed, nanowires being electrostatically glued on the tungsten tip, their initial positions are generally not symmetrical [7].

Note that simulations of articulated models have been widely used as an aid in the study of their continuous flexible counterparts in the field of dynamics [10]. In accordance with the physical problem and the purpose of the paper, this two degrees-offreedom model is expected to be sufficient to capture the essential dynamical features of the continuous system (geometrical nonlinearities, instability mechanism, self-oscillations). Furthermore, since most of the methods for analysing nonlinear systems are practicable only for low-dimensional systems, we follow the tendency to study a simplified discretization of the continuous system.

\subsection{Electrostatic model}

In the field emission configuration (Fig. 1), the applied $D C$ voltage $V$ leads to an electrical potential difference $U$ between the nanowire and the Ultra High Vacuum environment. Thereby, external electrostatic forces will act on the two segments of our bi-articulated nanostructure which behaves as one armature of a capacitor. According to the FE configuration (dimensions involved, Ultra High Vacuum chamber experiments), we consider that electrostatic interactions are an order of magnitude larger than other physical phenomena such as gravitational or Van der Waals forces, or Casimir and thermal effects. Electrostatic considerations are accordingly the only ones taken into account.

The electrostatic problem consists in the determination of the electric potential field $\mathfrak{U}$ in the vacuum domain under boundary conditions [13]. Under the assumptions of static potentials and no electrical charges inside the domain [14], this electrostatic problem can be reduced to the Laplace equation

$\Delta \mathfrak{U}=0$.

The unique solution of Laplace's equation must satisfy the Dirichlet boundary conditions imposed by the physical situation sketched in Fig. 2 b. A constant potential $U_{0}$ is imposed on the base $S_{0}$, the metallic structure composed of the tungsten tip support and the nanowire. The top surface $S_{1}$ is raised to a potential $U_{1}$ in order to simulate the voltage $U=U_{1}-U_{0}$ between the nanowire apex and its environment. Finally, we impose a linear evolution between $U_{0}$ and $U_{1}$ on the lateral face $S_{2}$. Boundaries $S_{1}$ and $S_{2}$ are chosen far enough from the nanowire to fulfil the hypothesis of a semi-infinite dielectric medium.

Once the electric potential $\mathfrak{U}$ is defined in the whole domain, the electric field is easily deduced [13] according to

$E=-\nabla \mathfrak{H}$.

Under the classical requirement that the electric field be everywhere perpendicular to the surface of the conductor, the electrostatic forces can be computed from the field at the surface of the nanowire following

$\underline{F}=\frac{1}{2} \varepsilon_{0} S \underline{E} .(\underline{E}, \underline{n})$,

where $\varepsilon_{0}=8.85 \times 10^{-12} \mathrm{~F} / \mathrm{m}$ is the permittivity of vacuum and $\underline{n}$ is the outer normal vector of the nanowire surface $S$. From an electrical point of view, the capacity between the nanowire and its environment derives simply [13] from the electric field $\underline{E}$ following

$C=\frac{\int_{S} 2 \varepsilon_{0} E \cdot \underline{n} \cdot \underline{d S}}{U_{1}-U_{0}}$

A last electrical variable provided by the electrostatic problem is the field enhancement factor $\beta$ illustrating the nanowire tip effect and involved in the expression of the FE current. Here, $\beta$ is the ratio between the electric field magnitude at the emitter apex and the electrical potential difference $U$ and reads

$\beta=\frac{E(W) \cdot \underline{n}}{U_{1}-U_{0}}$,

where $W$ is the point at the nanowire very end considered to be the emission surface (Fig. 2 b). The boundary value problem (2) is related to the geometrical configuration of the frontier $S_{0}$. According to the chosen kinematics, all the electrostatic quantities depicted in this part depend on the nanowire motion, characterized by the generalized coordinates $q_{1}$ and $q_{2}$. Moreover the electrostatic forces $F$ depend on potential difference $U$ and may also be written as $\left.\overline{F(} q_{1}, q_{2}, U\right)$. The electrical quantities $C$ and $\beta$ actually do not dependent on $U$ since they are expressed in the form $-\nabla \mathfrak{U} / U$ and read $C\left(q_{1}, q_{2}\right)$ and $\beta\left(q_{1}, q_{2}\right)$. a

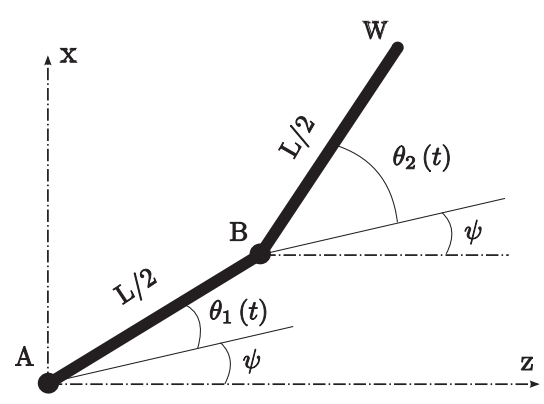

b

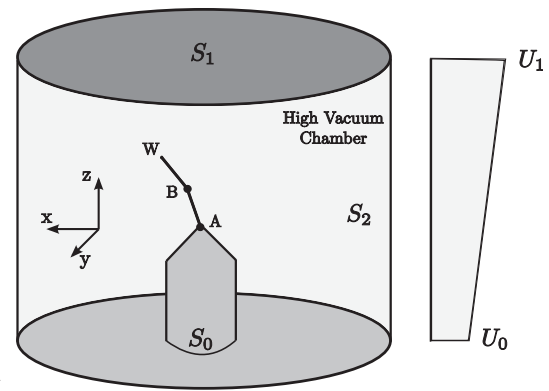

Fig. 2. Cantilevered bi-articulated nanowire immersed in electrostatic field. (a) Kinematics of the nanowire. (b) Sketch of the electrostatic problem. 


\subsection{Mechanical model}

We may now introduce the equations governing the motion of the cantilevered nanowire in field emission. The diagram in Fig. 3a illustrates the mechanical properties of the discrete model: $m$ is the nanowire mass per unit length, the constants $k_{1}$ and $k_{2}$ are the rotational stiffnesses of the rotational springs while $\mu$ characterizes the viscous damping applied to the angular velocities $\dot{q}_{1}$ and $\dot{q}_{2}$. The quantities $\underline{F}_{1}$ and $\underline{F}_{2}$ are the resultants of the electrostatic forces $F$ respectively on the first and second bar. They are defined by the previous electrostatic problem and also depend on the mechanical and electrical variables $q_{1}, q_{2}$ and $U$.

In order to express the governing equations directly in terms of the chosen generalized coordinates, we use the Lagrangian Formalism $[15,16]$. The system being nonconservative due to friction and electrostatic forces, we use the virtual power principle. The kinetic energy of the nanowire is naturally expressed in terms of the generalized coordinates $q_{1}(t)$ and $q_{2}(t)$ as

$\mathcal{T}=\frac{1}{2} m\left[\frac{L^{3}}{6} \dot{q}_{1}^{2}+\frac{L^{3}}{24} \dot{q}_{2}^{2}+\frac{L^{3}}{8} \dot{q}_{1} \dot{q}_{2} \cos \left(q_{1}-q_{2}\right)\right]$.

By introducing the two geometrically admissible angular velocities $\delta \dot{q}_{1}$ and $\delta \dot{q}_{2}$, the virtual power of acceleration quantities is simply obtained from the Lagrange formulae

$\mathcal{A}\left(\delta \dot{q}_{j}\right)=\left[\frac{d}{d t}\left(\frac{\partial \mathcal{T}}{\partial \dot{q}_{j}}\right)-\frac{\partial T}{\partial q_{j}}\right] \delta \dot{q}_{j} \quad$ for $j=1,2$.

We now express the virtual power done by the external forces for the virtual angular velocities $\delta \dot{q}_{j}$. We distinguish then between three contributions done respectively by the restoring elastic moments of the rotational springs $\mathcal{P}_{e 1}$, the restoring torque of viscous dampers $\mathcal{P}_{e 2}$ and the electrostatic forces $\mathcal{P}_{e 3}$. They read

$\mathcal{P}_{e 1}=-k_{1}\left(q_{1}-\psi\right) \delta \dot{q}_{1}-k_{2}\left(q_{1}-q_{2}\right) \delta \dot{q}_{1}-k_{2}\left(q_{2}-q_{1}\right) \delta \dot{q}_{2}$,

$\mathcal{P}_{e 2}=-c \dot{q}_{1} \delta \dot{q}_{1}-c \dot{q}_{2} \delta \dot{q}_{2}$

$\mathcal{P}_{e 3}=\left(\mathfrak{M}_{1 A}+\mathfrak{M}_{2 A}\right) \delta \dot{q}_{1}+\mathfrak{M}_{2 B} \delta \dot{q}_{2}$.

According to our kinematics, the virtual power $\mathcal{P}_{e 3}$ is expressed in terms of the moments of the electrostatic forces about the two articulations which depend on the variables $q_{1}, q_{2}$ and $U$. While $\mathfrak{M}_{1 A}$ and $\mathfrak{M}_{2 A}$ are respectively the torques of the resultants $F_{1}$ and $F_{2}$ about the first articulation $A, \mathfrak{M}_{2 B}$ is the moment of $F_{2}$ about the second articulation $B$.

Finally, the principle of virtual power $\mathcal{A}\left(\delta \dot{q}_{i}\right)=\mathcal{P}_{e}\left(\delta \dot{q}_{i}\right)+\mathcal{P}_{i}\left(\delta \dot{q}_{i}\right)$ for each virtual variations $\delta \dot{q}_{i}$ leads to the nonconservative Lagrange's equations. Given that $\mathcal{P}_{i}\left(\delta \dot{q}_{i}\right)=0$ in our system, the nonlinear governing equations of the initially tilded bi-articulated nanowire read

$$
\begin{aligned}
\frac{1}{6} m L^{3} \ddot{q}_{1} & +\frac{1}{16} m L^{3} \ddot{q}_{2} \cos \left(q_{1}-q_{2}\right)+\frac{1}{16} m L^{3} \dot{q}_{2}^{2} \sin \left(q_{1}-q_{2}\right)+\mu \dot{q}_{1} \\
& +k_{1}\left(q_{1}-\psi\right)+k_{2}\left(q_{1}-q_{2}\right)-\mathfrak{M}_{1 A}-\mathfrak{M}_{2 A}=0, \\
\frac{1}{24} m L^{3} \ddot{q}_{2} & +\frac{1}{16} m L^{3} \ddot{q}_{1} \cos \left(q_{1}-q_{2}\right)-\frac{1}{16} m L^{3} \dot{q}_{1}^{2} \sin \left(q_{1}-q_{2}\right)+\mu \dot{q}_{2} \\
& +k_{2}\left(q_{2}-q_{1}\right)-\mathfrak{M}_{2 B}=0,
\end{aligned}
$$

where $\mathfrak{M}_{1 A}, \mathfrak{M}_{2 A}$ and $\mathfrak{M}_{2 B}$ depend on the generalized coordinates $q_{1}, q_{2}$ and the electrical voltage $U$. Eqs. (10a), (9b) define the mechanical model.

\subsection{Electrical model}

In field emission configuration, the electrons extraction leads to a FE current $I_{e}(t)$ flowing inside the conducting nanowire. As a consequence, the voltage $U$ between the cantilevered nanostructure and its environment governing the electrostatic moments is not given by the applied DC voltage $V[9]$. Actually, the voltage $U(t)$ is determined by the experimental physical constraints, coming down to the electrical circuit sketched in Fig. 3 b where notably, $V$ is the applied DC voltage, $R$ the nanowire resistivity, $C$ the nanowire capacitance, $I_{c}(t)$ the capacitor current and $I_{e}(t)$ the FE current.

As for the mechanical governing equation, the differential equation of the electrical circuit is obtained through the Lagrangian formalism. Considering the charge $q_{3}$ flowing inside the circuit as a generalized coordinate, so that $\dot{q}_{3}=I$ is simply the electric current, the electric power done for the virtual current $\delta \dot{q}_{3}$ is noted

$\mathcal{P}_{e l}=-V \delta \dot{q}_{3}+R I \delta \dot{q}_{3}+U \delta \dot{q}_{3}$

From this point, it is more convenient to express $\mathcal{P}_{e l}$ in terms of $U(t)$ involved in the mechanical equation (10) through the electrostatic moments. According to Kirchhoff's current law, the electric current flowing in the nanowire reads $I=I_{c}+I_{e}$ where $I_{c}$ and $I_{e}$ are given by

$I_{c}\left(q_{1}, q_{2}, U\right)=\frac{d(C U)}{d t}=C \dot{U}+U\left(\frac{\partial C}{\partial q_{1}} \dot{q}_{1}+\frac{\partial C}{\partial q_{2}} \dot{q}_{2}\right)$,

$I_{e}\left(q_{1}, q_{2}, U\right)=H \beta^{2} U^{2} e^{-G / \beta U}$.

The capacitor current $I_{c}$ is given by (12a) where the second term is due to the dependence of the capacitance on the nanowire position pointed out in the previous section. The current $I_{e}$ is given by the field emission theory [6] and Eq. (12b) is the Fowler-Nordheim formula where constants $H$ and $G$ are empirical and the field enhancement factor $\beta$ is defined by the previous electrostatic problem. For the cantilevered nanoemitter in field emission, $I_{e}$ and $I_{c}$ depend not only on the voltage $U$ but on the nanowire position $q_{1}$ and $q_{2}$ through the electrostatic variables $\beta$ and $C$. a

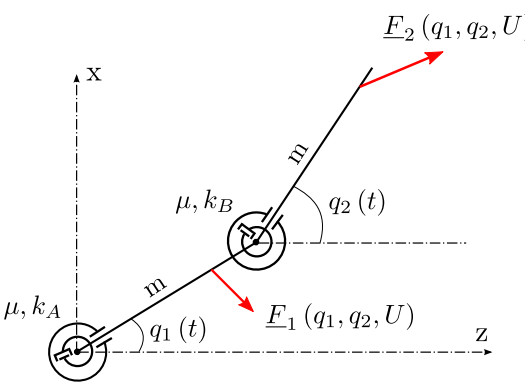

b

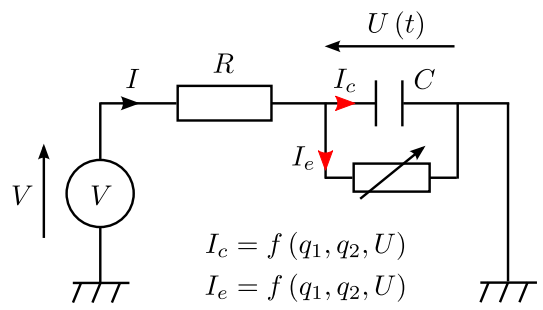

Fig. 3. Sketches of the NanoElectroMechanical System. (a) Mechanical contribution. (b) Electric contribution. 
Finally, replacing the relations (12) in the virtual electric power (11) and applying the virtual power principle for each virtual variations $\delta \dot{q}_{3}$ which is simply $\mathcal{P}_{e l}=0$, we obtain

$R C \dot{U}+R U\left(\frac{\partial C}{\partial q_{1}} \dot{q}_{1}+\frac{\partial C}{\partial q_{2}} \dot{q}_{2}\right)+R H \beta^{2} U^{2} e^{-G / \beta U}+U-V=0$

where $C$ and $\beta$ depend on $q_{1}, q_{2}$ according to the previous electrostatic relations (5) and (6). This nonlinear equation governs the voltage $U(t)$ which is the third generalized coordinates necessary to define the configuration of our electromechanical system at each time $t$.

\subsection{Dimensionless form}

The dimensionless electrical potential $\mathfrak{u}=\left(\mathfrak{H}-U_{0}\right) /\left(U_{1}-U_{0}\right)$ is obtained by computing the Laplace equation

$\Delta \mathfrak{u}=0$

with the Dirichlet boundary conditions such as $\mathfrak{u}=0$ on the base $S_{0}, \mathfrak{u}=1$ on the top surface $S_{1}$ and so that a linear evolution between 0 and 1 is imposed on the lateral face $S_{2}$ (Fig. 2b). The dimensionless electrical field $e$ is the opposite gradient of $\mathfrak{u}$. The electrostatic moments resulting from the dimensionless electrostatic problem are noted $\mathfrak{M}_{0}$ and only depend on the generalized coordinates $q_{1}$ and $q_{2}$. Let $U_{\text {ref }}$ be the reference voltage above which the Fe current $I_{e}$ given by (12b) is no more negligible. We introduce the constants $C_{r e f}$ and $\beta_{\text {ref }}$ which are respectively the capacitance and amplification factor of the nanostructure in its symmetrical position $\left(q_{1}, q_{2}\right)=(0,0)$. Using $\omega=\sqrt{k_{1} / m L^{3}}$ as a reference frequency for the mechanical oscillator, we define the dimensionless variables:

$\tau=\omega t, \quad Q=\frac{\sqrt{k_{1} m L^{3}}}{c}, \quad u=\frac{U}{U_{\text {ref }}}, \quad a_{k}=\frac{k_{2}}{k_{1}}, \quad r=\omega R C_{r e f}$,

$h=R H \beta_{r e f}^{2} U_{r e f}, \quad g=\frac{-G}{\beta_{r e f} U_{r e f}}, \quad i_{e}=h b^{2} u^{2} e^{g / b u}$,

$\mathfrak{m}=\mathfrak{M}_{0} \frac{U_{\text {ref }}^{2}}{k_{1}}, \quad c=\frac{C}{C_{\text {ref }}}, \quad b=\frac{\beta}{\beta_{\text {ref }}}$

In particular, $Q$ is the nanowire quality factor and $r$ the ratio between the electrical and mechanical time constants. The quantity $i_{e}$ is the dimensionless form of the FE current $I_{e}$ and $\mathrm{m}$ is the dimensionless electrostatic moment. After simple calculations, Eqs. (10) and (13) may be re-written in dimensionless form as

$$
\begin{aligned}
\ddot{q}_{1} & +\frac{3}{8} \ddot{q}_{2} \cos \left(q_{1}-q_{2}\right)+\frac{3}{8} \dot{q}_{2}^{2} \sin \left(q_{1}-q_{2}\right)+\frac{6}{Q} \dot{q}_{1}+6\left(q_{1}-\psi\right) \\
& +6 a_{k}\left(q_{1}-q_{2}\right)-6\left[\mathrm{~m}_{1 A}+\mathrm{m}_{2 A}\right] u^{2}=0,
\end{aligned}
$$

$$
\begin{aligned}
\ddot{q}_{2} & +\frac{3}{2} \ddot{q}_{1} \cos \left(q_{1}-q_{2}\right)-\frac{3}{2} \dot{q}_{1}^{2} \sin \left(q_{1}-q_{2}\right)+\frac{24}{Q} \dot{q}_{2} \\
& +24 a_{k}\left(q_{2}-q_{1}\right)-24 m_{2 B} u^{2}=0,
\end{aligned}
$$

$r c \dot{u}+r u\left(c_{, 1} \dot{q}_{1}+c_{, 2} \dot{q}_{2}\right)+h b^{2} u^{2} e^{g / b u}+u-v=0$,

where $c_{j}=\partial c / \partial q_{j}$ for $j=1,2$. Eqs. (16) are the nonlinear dimensionless governing equations of a cantilevered bi-articulated nanowire in field emission. The dynamic behaviour of this NanoElectroMechanical System is determined by computing unknown generalized coordinates $q_{1}, q_{2}$ and $u$ at any time $t$ knowing that all the other quantities are defined. Indeed, variables $m_{1 A}\left(q_{1}, q_{2}\right), \quad m_{2 A}\left(q_{1}, q_{2}\right), \quad m_{2 B}\left(q_{1}, q_{2}\right), \quad c\left(q_{1}, q_{2}\right)$ and $b\left(q_{1}, q_{2}\right)$, responsible for the electromechanical interactions, are determined after solving the independent dimensionless electrostatic problem (14). As for the remaining electrical or mechanical parameters, they are directly derived from experimental data and presented in the following section.

\section{Values of parameters}

In this section, the mechanical and electrical model parameters are defined in order to simulate and study the self-oscillations of a nanowire in field emission. It will also specify the order of magnitude of the involved physical quantities.

\subsection{Electromechanical parameters}

The dimensions and the material properties of the investigated nanostructures are completely given by the vapour-solid growth mechanism of the silicon carbide ( $\mathrm{SiC}$ ) nanowires. According to their typical experimental aspect ratio, we assume a length $L=10 \mu \mathrm{m}$ and a circular cross section with diameter $D=200 \mathrm{~nm}$ (giving a area moment of inertia $I_{n}=\pi D^{4} / 64$ ). The nanowire material being almost equivalent to pure carbon, the density is $\rho=3200 \mathrm{~kg} / \mathrm{m}^{3}$ and leads to a mass per unit length expressed as $m=\rho \pi(D / 2)^{2}=1 \times 10^{-10} \mathrm{~kg}$. Young's modulus $E$ is determined by field emission [7] and is taken as $E=400 \mathrm{GPa}$. At room temperature and in an Ultra High Vacuum chamber, the mechanical quality factor of a singly clamped nanowire can reach 160000 [2]. In our simulations, we choose a classic value $Q$ $=20000$. The Fowler-Nordheim constants introduced in (12b) are experimentally found as $H=2.73 \times 10^{-24} \mathrm{~A} \mathrm{~m}^{2} \mathrm{~V}^{-2}$ and $G=4.4145 \times 10^{10} \mathrm{mV}^{-1}[9]$. Finally, the SiC nanowires are highly resistive and their electrical resistance can be taken as $R=10^{10} \Omega$.

Coming back to the discrete model determined in the previous section, one has to adapt the given experimental quantities to the actual parameters necessary in the governing equation (16). The electric constants $R, H$ and $G$ can directly be used in the model, and the same is true for the mechanical parameters $L$ or $Q$. However, the springs stiffnesses and the rigid bars mass have to model the mechanical behaviour of the continuous structure characterized by $E, I_{n}$ and its mass. Because of our lowdimensional discretization, we will not capture all dynamical experimental features. In this paper, we assume the mass per unit length $m$ to be equal to the experimental one. In order to keep a good static model, we impose that the static deflection of the cantilevered nanowire under an end load $F_{0}$ is equivalent for the continuous and its discrete counterpart. According to [17], it comes simply

$\frac{F_{0} L^{3}}{3 E I}=\frac{L}{2} \frac{F_{0} L}{k_{1}}+\frac{L}{2} \frac{F_{0} L}{2 k_{2}}$.

In order to ensure consistency in the dynamic model, we constrain the ratio between the natural frequencies of the clamped-free beam first two modes to be equivalent in the discrete and continuous model. While this ratio reads $\omega_{2} / \omega_{1}=6.3$ in the continuous case [17], the discrete case is obtained by solving the set of equations

$\left[\begin{array}{cc}\frac{1}{6} m L^{3} & \frac{1}{16} m L^{3} \\ \frac{1}{16} m L^{3} & \frac{1}{24} m L^{3}\end{array}\right]\left(\begin{array}{l}\ddot{q}_{1} \\ \ddot{q}_{2}\end{array}\right)+\left[\begin{array}{cc}k_{1}+k_{2} & -k_{2} \\ -k_{2} & k_{2}\end{array}\right]\left(\begin{array}{l}q_{1} \\ q_{2}\end{array}\right)=\left(\begin{array}{l}0 \\ 0\end{array}\right)$.

Eq. (18) is directly derived from the linearization of the mechanical equations (10) when no damping, no initial tilting angle $\psi$ and no electrostatic forces are considered. By simply respecting the required ratio between the eigenfrequencies of 
Eqs. (18), we obtain the desired parameter which are in our specific case $k_{1}=2.36 \mathrm{EI} / L$ and $k_{2}=2.06 \mathrm{EI} / L$., i.e. a stiffness ratio $a_{k}=0.873$.

\subsection{Electrostatic variables}

The electrostatic variables $c, b$ and $m$ are not obtained directly from the experimental data but through the electrostatic problem given in Section 2.2 and they depend also strongly on the nanowire aspect ratio $L / D$. The determination of these parameters and their related quantities such as $r, U_{\text {ref, }} \beta_{\text {ref }}$ and $C_{\text {ref, }}$, are described in the following.

The electrostatic problem sketched in Fig. 2 and accounted for the dimensionless Laplace equation (14) is computed using the finite element software Cast3m [18]. Solving this equation for different sets of generalized angular displacements $\left(q_{1}, q_{2}\right)$ yields a discrete map of the dimensionless electrostatic quantities in the $\left(q_{1}, q_{2}\right)$ space. A polynomial regression is then sufficient to define the continuous field enhancement factor $b\left(q_{1}, q_{2}\right)$, the electrical capacitance $c\left(q_{1}, q_{2}\right)$ and electrostatic moments $\mathrm{m}\left(q_{1}, q_{2}\right)$ that are necessary for the further numerical analysis of the governing equations.

The vacuum chamber and the boundary surfaces $S_{0}, S_{1}$ and $S_{01}$ are generated using 3D elements (20 nodes hexahedral) and are represented in Fig. 4. According to our kinematics, the cantilevered nanowire of length $L$ is modelled by two cylindrical bars with diameter $D$ making an absolute angle $q_{1}$ and $q_{2}$ with the symmetrical configuration (Fig. 4b). The nanowire apex is represented by a perfect semi-sphere oriented following the upper bar direction. Due to the large scale ratio between the structure and its environment, electrostatic phenomena will take place mostly in the vicinity of the nanowire. As a consequence, the regular mesh has to be refined nearby the structure but relaxed far from it to keep reasonable computation times. Finally, the solution of the dimensionless Laplace equation (14) is discretized with quadratic shape functions in order to correctly model the electrostatic field evolution near the nanowire apex. The system of linear equations arising from the approximation of (14) is solved using Crout method [19].

A typical electric potential (for $q_{1}=10^{\circ}$ and $q_{2}=-4^{\circ}$ ) is shown in Fig. $5 \mathrm{a}$ for a cross-section of the vacuum chamber in the vicinity of the bi-articulated nanowire. Most of the change in the scalar field $\mathfrak{u}$ takes place close to the nanowire. As a consequence, the electric field $e=-\nabla \mathfrak{u}$, is localized all around the structure (Fig. 5 b). Due to the large value of the aspect ratio, $e$ is concentrated at its apex as expected (tip effect).

According to the dimensionless form of the electrostatic problem, the field enhancement factor $\beta$ is just the magnitude of the electric field at the pole of the semi-sphere while the electric capacitance $C$ is the total charge of electrons on the nanowire given by (5). The continuous functions $b\left(q_{1}, q_{2}\right)$ and $c\left(q_{1}\right.$, $q_{2}$ ) are obtained by applying a second order polynomial regression to their discrete value computed for hundred values of $\left(q_{1}, q_{2}\right)$ in the range $\left[-10^{\circ}, 10^{\circ}\right]$. They read

$b\left(q_{1}, q_{2}\right)=-0.058 q_{1}^{2}-0.074 q_{2}^{2}+0.019 q_{1} q_{2}$,

$c\left(q_{1}, q_{2}\right)=-0.105 q_{1}^{2}-0.06 q_{2}^{2}+0.082 q_{1} q_{2}$

given that $\beta_{\text {ref }}=1.032 \times 10^{7} \mathrm{~m}^{-1}$ and $C_{\text {ref }}=5.192 \times 10^{-17} \mathrm{~F}$ for the symmetrical position $\left(q_{1}, q_{2}\right)=(0,0)$. These electrical functions are maximal for the symmetrical position $(0,0)$ and decrease quadratically when the nanowire moves away from it. Note that for practical purpose, the nanowire apex is not perfectly smooth
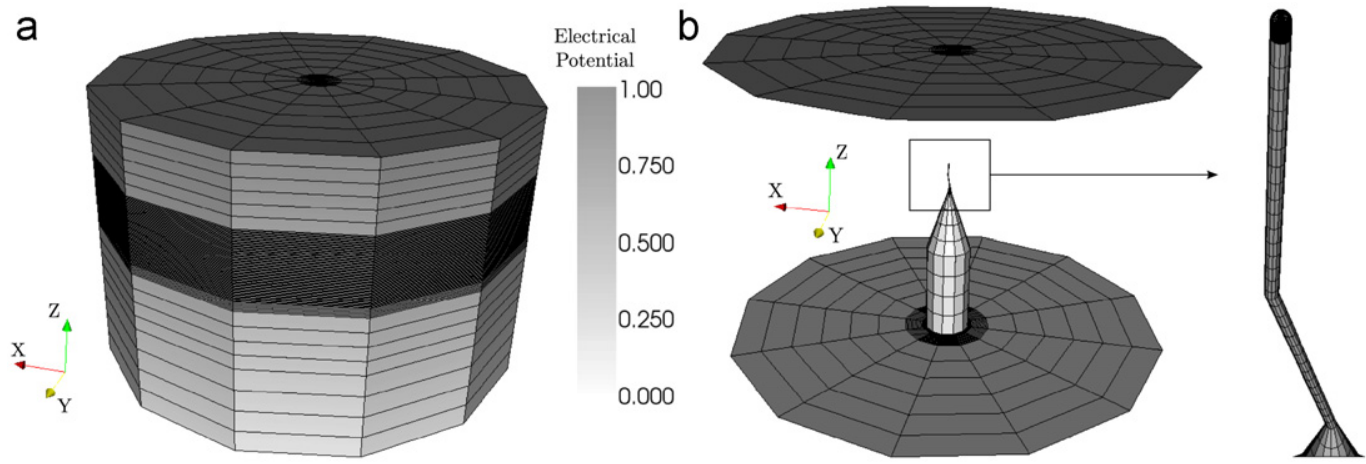

Fig. 4. 3D Finite element model of the electrostatic boundaries. (a) Outside view of the electrostatic box. (b) Nanowire: $q_{1}=10^{\circ}$ and $q_{2}=-4$.

a

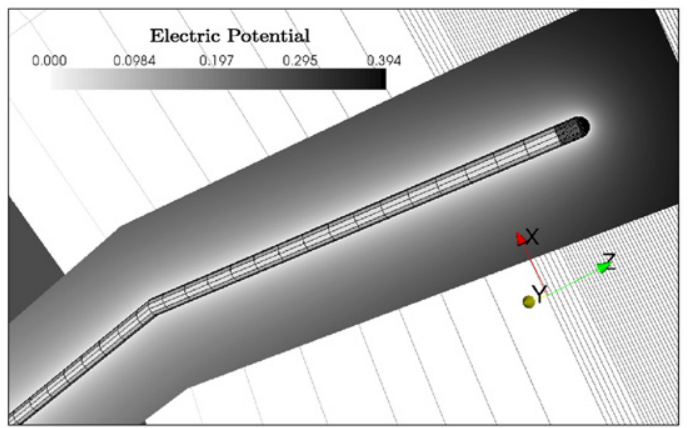

b

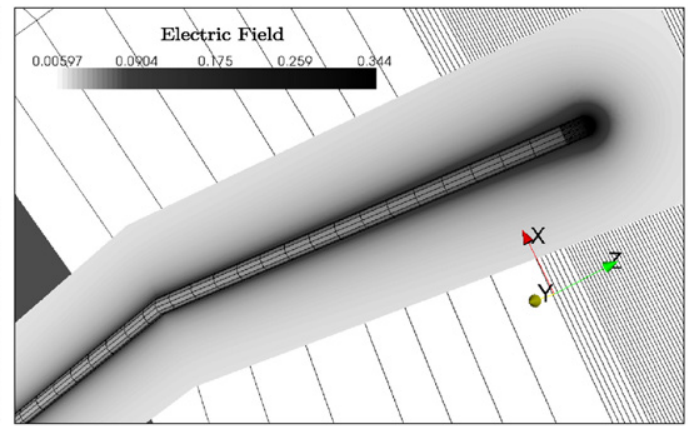

Fig. 5. Electrostatic fields nearby the nanowire for $q_{1}=10^{\circ}$ and $q_{2}=-4^{\circ}$. (a) Evolution of electric potential. (b) Evolution of electric field magnitude. 

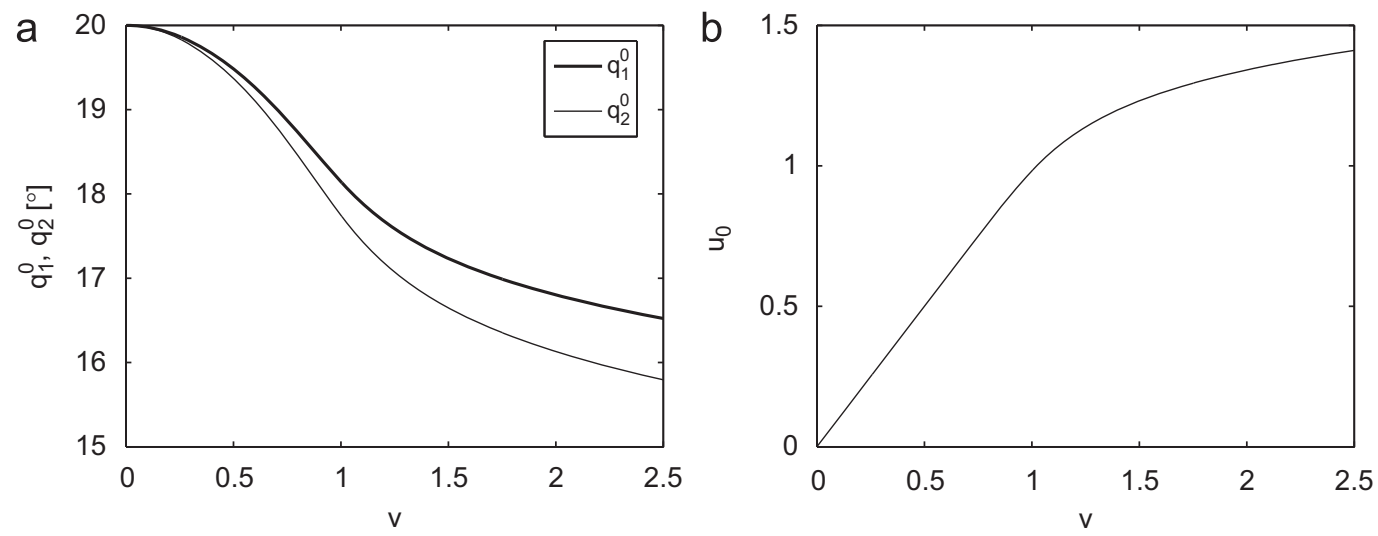

Fig. 6. Evolution of the fixed points $\left(q_{1}^{0}, q_{2}^{0}, u_{0}\right)$ against $v$ for $\psi=20^{\circ}$. (a) Mechanical static equilibrium. (b) Electrical static equilibrium.

but made up of several protrusions which are the real field emitters. According to classical electrostatic theory [13], $\beta_{\text {ref }}$ has also been multiplied by 3 to take into account the electric field amplification due to a hemispherical protrusion. Once these quantities computed, the ratio of time constants reads simply $r=\omega R C_{\text {ref }}=4.458$. The voltage reference $U_{\text {ref }}$ corresponds to the emergence of the FE current $i_{e}$ inside the emitter. For practical purposes, it is chosen so that $i_{e}>0.01$ when $U>U_{\text {ref }}$ and is $U_{r e f}=$ $400 \mathrm{~V}$ in our case. Below this reference value, the FE current given by the Fowler-Nordheim formula (12b) is negligible due to the exponential term $e^{-G / \beta U}$. The shape of the dimensionless current $I_{e}$ in the $(q, u)$ space is given in Appendix A (Fig. 10).

The electrostatic forces derive from the electric field $e$ according to (4). Two particular features can be made out. First, the tip effect pointed out in Fig. 5b introduces strong partial following [20] pulling forces at the nanowire apex. Second, the symmetry of the electric field being broken by the leaning nanowire, the resultant electrostatic forces are restoring forces trying to bring the structure back to its symmetrical position. The electrostatic moments $m_{1 A}, m_{2 A}$ and $m_{2 B}$ about the two articulations arise from the vector product between the electrostatic forces and their distance with the considered articulation. The coefficients of the second order polynomial regressions of the discrete value of $m_{1 A}, m_{2 A}$ and $m_{2 B}$ computed in the $\left(q_{1}, q_{2}\right)$ space are given by

$\mathrm{m}_{1 A}\left(q_{1}, q_{2}\right)=0.026 q_{1}-0.051 q_{2}$

$\mathrm{m}_{2 A}\left(q_{1}, q_{2}\right)=-0.117 q_{1}+0.054 q_{2}$,

$\mathrm{m}_{2 B}\left(q_{1}, q_{2}\right)=0.008 q_{1}-0.029 q_{2}$.

The evolution of the moments is simply linear and their signs are in agreement with the "restoring forces" properties. Moreover, the magnitude of the moments decreases with the generalized coordinates $\left(q_{1}, q_{2}\right)$ down to the symmetrical position $(0,0)$ where they cancel.

\section{Numerical results}

\subsection{Computation of the static position}

The static equilibrium of the bi-articulated nanowire, later called the base state, depends on the constant applied voltage $V$ and is obtained by computing the fixed points $\left(q_{1}^{0}, q_{2}^{0}, u_{0}\right)$ of system (16):

$\left(q_{1}^{0}-\psi\right)+a_{k}\left(q_{1}^{0}-q_{2}^{0}\right)-\left[\mathfrak{m}_{1 A}+\mathfrak{m}_{2 A}\right] u_{0}^{2}=0$,

$a_{k}\left(q_{2}^{0}-q_{1}^{0}\right)-m_{2 B} u_{0}^{2}=0$

$v-u_{0}-h b^{2} u_{0}^{2} e^{\left(g / b u_{0}\right)}=0$.

In this nonlinear set of equation, the dimensionless electrical and mechanical quantities have been defined in Section 3 where the continuous functions $b\left(q_{1}^{0}, q_{2}^{0}\right)$ and $m\left(q_{1}^{0}, q_{2}^{0}\right)$ are given by (19) and (20). The base state branch [21] deriving continuously from the base state $\left(q_{1}^{0}, q_{2}^{0}, u_{0}\right)=(\psi, \psi, 0)$ at $v=0$, is determined by progressively increasing the control parameter $v$. The problem $f\left(q_{1}^{0}, q_{2}^{0}, u_{0}\right)=0$ is solved using Newton-Raphson method for each step $v$ with the initial guess coming from the previous step.

For an initial tilting $\psi=20^{\circ}$, we plot the evolution of fixed points $\left(q_{1}^{0}, q_{2}^{0}, u_{0}\right)$ against $v$ in Fig. 6 . Two distinct behaviours can be clearly removed from the smooth branches. For a constant applied voltage $V$ lower than the voltage reference $V_{\text {ref }}$ (i.e. $v<1$ ), the FE current $I_{e}$ given by the Fowler-Nordheim formula (12b) is negligible (no emission). Thus, according to the electrical equation (21c), the voltage at the nanowire apex reads directly $u_{0}=v$ (Fig. 6b). The restoring electrostatic moments being proportional to $u_{0}^{2}$, the bi-articulated nanowire is almost quadratically coming back to its symmetrical position (Fig. 6a).

In emission configuration, i.e. for $v>1$, the FE current $I_{e}$ is no longer negligible and increases exponentially with $u_{0}$, following the Fowler-Nordheim equation. Due to the nanowire electrical resistance, a supplementary voltage behaving exponentially as $I_{e}$ appears inside the electrical circuit model by (21c). As a consequence, the voltage $u_{0}$ between the nanowire and its environment saturates when increasing $v$ (Fig. 6 b). The electrostatic moments saturate as well and the same is true for the evolution of the generalized coordinates $\left(q_{1}, q_{2}\right)$ (Fig. 6a).

\subsection{Stability of the static solutions}

We consider the perturbation expansions in the form $q(\tau)=q_{0}+\varepsilon \tilde{q}(\tau)$ and $u(\tau)=u_{0}+\varepsilon \tilde{u}(\tau)$. Substituting these expansions into the dimensionless governing Eq. (16) and equating the first power of $\varepsilon$, we express the linearized governing equation around the static equilibrium $\left(q_{1}^{0}, q_{2}^{0}, u_{0}\right)$

$\ddot{\tilde{q}}_{1}+\frac{3}{8} \ddot{\tilde{q}}_{2} \cos \left(q_{1}^{0}-q_{2}^{0}\right)+\frac{6}{Q} \dot{\tilde{q}}_{1}+6\left(\tilde{q}_{1}-\psi\right)+6 a_{k}\left(\tilde{q}_{1}-\tilde{q}_{2}\right)$ 


$$
-6 u_{0}^{2} \sum_{j=1}^{2}\left[\mathrm{~m}_{1 A, j}+\mathrm{m}_{2 A, j}\right] \tilde{q}_{j}-12 u_{0}\left[\mathfrak{m}_{1 A}+\mathrm{m}_{2 A}\right] \tilde{u}=0
$$

$$
\begin{aligned}
& \ddot{\tilde{q}}_{2}+\frac{3}{2} \ddot{\tilde{q}}_{1} \cos \left(q_{1}^{0}-q_{2}^{0}\right)+\frac{24}{Q} \dot{\tilde{q}}_{2}+24 a_{k}\left(\tilde{q}_{2}-\tilde{q}_{1}\right)-48 u_{0} \mathrm{~m}_{2 B} \tilde{u} \\
& -24 u_{0}^{2} \sum_{j=1}^{2} \mathrm{~m}_{2 B, j} \tilde{q}_{j}=0
\end{aligned}
$$

$$
r c \dot{\tilde{u}}+r u_{0} \sum_{j=1}^{2} c_{j} \dot{\tilde{q}}_{j}+\left(1+i_{e, \tilde{u}}\right) \tilde{u}+\sum_{j=1}^{2} i_{e, j} \tilde{q}_{j}=0 .
$$

The dimensionless FE current $i_{e}$, the electrical capacitance $c$ and electrostatic moments $m$ are continuous functions of $\left(q_{1}^{0}, q_{2}^{0}\right)$ according to (19) and (20) obtained from the electrostatic problem. Their partial derivatives with respect to $\tilde{q}_{j}$ are denoted by the subscript ()$_{j}$ and defined at $\left(q_{1}^{0}, q_{2}^{0}\right)$. In particular, derivatives of the FE current are determined with $b\left(q_{1}^{0}, q_{2}^{0}\right)$ given in (19) following

$\left.i_{e, j}=\frac{i_{e}^{0}}{b^{2}}\left(b_{j} b-g \frac{b_{j}}{u_{0}}\right), \quad i_{e, \tilde{u}}=i_{e}^{0} \quad \frac{2}{u_{0}}-\frac{g}{b u_{0}^{2}}\right), \quad i_{e}^{0}=i_{e}\left(q_{1}^{0}, q_{2}^{0}, u_{0}\right)$

and where the subscript ()$_{, \tilde{u}}$ denotes the derivative with respect to $\tilde{u}$.

Eq. (22) may be rewritten in the physical space $\underline{Z}(\tau)=\left[\begin{array}{lll}\tilde{q}_{1} & \tilde{q}_{2} & \tilde{u}\end{array}\right]^{T}$ in the more compact way

$\mathbb{M} \underline{Z}(\tau)+\mathbb{D} \underline{Z}(\tau)+\mathbb{K} \underline{Z}(\tau)=\underline{0}$,

where $\mathbb{M}, \mathbb{D}$ and $\mathbb{K}$ are respectively the mass, damping and stiffness matrix of the coupled system (22). The stability of the base state branches $\left(q_{1}^{0}, q_{2}^{0}, u_{0}\right)$ is reached by stating the perturbation $Z(\tau)$ in the form

$\underline{Z}(\tau)=\underline{\varphi e^{s \tau}}$ with $\underline{\varphi}=\left[\begin{array}{lll}\tilde{q}_{1}^{A} & \tilde{q}_{2}^{A} & \tilde{u}^{A}\end{array}\right]^{T}$,

where the characteristic exponent $s=\sigma+i \omega$ leads to the decay rate $\sigma$ and the dimensionless frequency $\omega$ of the eigenmode $\varphi$. For practical purpose, the eigenproblem arising from (24) is solved in the phase space $W(\tau)=\left[\begin{array}{lllll}\tilde{q}_{1} & \tilde{q}_{2} & \tilde{u} & \dot{\tilde{q}}_{1} & \dot{\tilde{q}}_{2}\end{array}\right]^{T}$ where (24) becomes

$\mathbb{B} \dot{W}(\tau)-\mathbb{A} W(\tau)=0$

and $\underline{W}(\tau)=\underline{\Phi} e^{s \tau}$ so that the computed eigenproblem reads

$[s \mathbb{B}-\mathbb{A}] \underline{\Phi}=\underline{0} \quad$ with $\underline{\Phi}=\left[\begin{array}{lllll}\tilde{q}_{1}^{A} & \tilde{q}_{2}^{A} & \tilde{u}^{A} & s \tilde{q}_{1}^{A} & s \tilde{q}_{2}^{A}\end{array}\right]^{T}$.
For each value of the control parameter $v$, the fixed points $\left(q_{1}^{0}, q_{2}^{0}\right.$, $u_{0}$ ) are computed using (21) so that the linearized governing equation (22) is completely defined. The eigenmodes $\varphi$ and their associated eigenvalues $s$ are then computed for each $v$ with (27).

For $v=0$, the electromechanical system (22) is totally uncoupled given that $u_{0}=i_{e}^{0}=0$. The first eigenmodes $\varphi_{1}$ and $\varphi_{2}$ are the mechanical modes of the bi-articulated nanowire of $\overline{t h e}^{2}$ form $\varphi=\left[\begin{array}{lll}\tilde{q}_{1}^{A} & \tilde{q}_{2}^{A} & 0\end{array}\right]^{T}$. These entities are respectively the classical first and second bi-articulated modes with natural frequencies $\omega_{1}$ and $\omega_{2}$ given in Section 3 and where the decay rates $\sigma_{1}$ and $\sigma_{2}$ are linked to the quality factor $Q$. The third eigenmode $\varphi_{3}$ is the electrical mode of the $R C$ circuit formed by the nanowire. This stationary mode $\left(\omega_{3}=0\right)$ is associated with the strong decay rate $\sigma_{3}=-1 / R C$.

When increasing $v$, the purely mechanical and purely electrical modes combine into electromechanical modes. The electrical contribution $\tilde{u}^{A}$ in the oscillating modes $\varphi_{1}$ and $\varphi_{2}$ grows progressively and the eigenvalues $s$ vary. In the same way, the mechanical contributions $\tilde{q}_{1}^{A}$ and $\tilde{q}_{2}^{A}$ increase in the stationary mode $\varphi_{3}$ but the decay rate $\sigma_{3}$ is strongly decreasing. Thus, the interesting physical phenomenon will be contained in the first mode shapes $\varphi_{1}$ and $\varphi_{2}$. In the following, only the natural frequencies and decay rate of these first two modes are investigated in order to determine the stability of the fixed points plotted in Fig. 6.

The evolution of the dimensionless eigenvalues $s$ against $v$ for a cantilevered bi-articulated nanowire in field emission is displayed in Fig. 7 for the situation of interest. The increase of the natural frequencies showed in Fig. 7a accounts for the strong electrostatic pulling coming from the tip effect in field emission (already expected in Section 3.2). As in Section 4.2 for the computation of fixed points, two distinct behaviours are observed depending on the applied voltage $V$ compared to the field emission reference voltage $U_{\text {ref. }}$. Indeed, the saturation of the voltage $u_{0}$ illustrated in Fig. $6 \mathrm{~b}$ and due to the FE current emergence is directly reflected in the electrostatic pulling forces, i.e. in the geometric stiffness.

According to the variation of the decay rate given in Fig. 7b, the first mode $\varphi_{1}$ becomes linearly unstable for a low voltage $v$, highlighting thereby the qualitative agreement between the bi-articulated nanowire modelling and the experimental observations made in [9]. As above, it is possible to define two domains in the decay rate evolution, separated by an inflexion point located at $v=1$, indicating that the field emission must be involved in the destabilization process. A simpler kinematic model (straight nanowire), given in Appendix A, may be used to approximate a

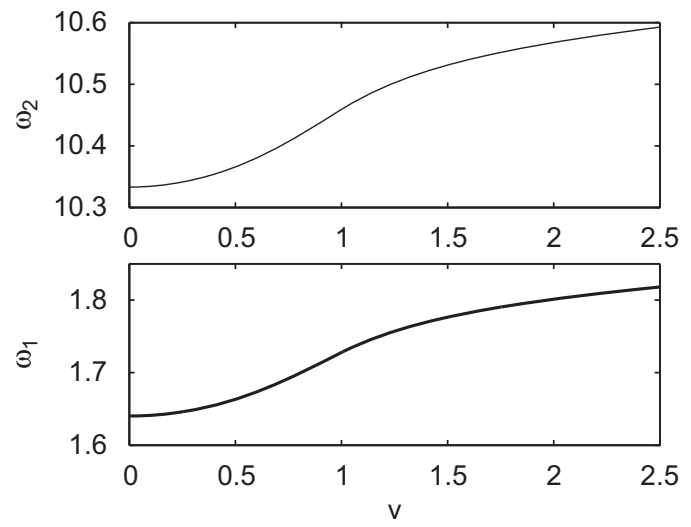

b

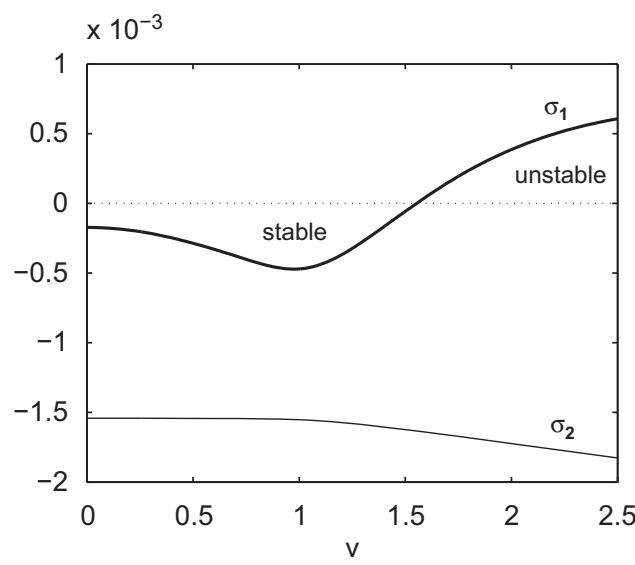

Fig. 7. Linear vibratory behaviour of the static equilibrium against $v$. (a) Evolution of the natural frequencies. (b) Evolution of the decay rates. 
this FE instability mechanism. Notably, the analytical form of $\sigma(v)$ points out the interplay of the electromechanical parameters triggering such phenomenon.

\subsection{Stability domain}

By computing the eigenproblem (27) for different set of dimensionless parameters, we obtain the stability map of the cantilevered bi-articulated nanowire in field emission. Fig. 8 shows the decay rate of the mode $\varphi$ in the dimensionless spaces $(v, \psi),(v, Q)$ and $(v, r)$. The white regions are related to $\sigma<0$ and are also referred as stability regions while the dark ones display the decay rates $\sigma>0$, i.e. the instability regions where the hue illustrates the magnitude of $\sigma$. The numerical parameters used in the computations are those defined in Section $3, \psi=20^{\circ}, Q=$ 20000 and $r=\omega R C_{\text {ref }}=4.458$.

Fig. 8a represents the stability map of the NanoElectroMechanical System when varying the initial angle $\psi$ and shows that a minimum angular imperfection is needed to trigger the instability. Actually, if the nanowire position $\left(q_{1}^{0}, q_{2}^{0}\right)$ is too close from its symmetrical position $(0,0)$, the FE current dependence on $\left(q_{1}, q_{2}\right)$ is not sufficient to counterbalance the stabilizing terms $i_{e, \tilde{u}}$ and $c_{j}$ given in Eq. (22). Indeed, for a given $u_{0}, i_{e}\left(q_{1}^{0}, q_{2}^{0}, u_{0}\right)$ is maximal at $\left(q_{1}^{0}, q_{2}^{0}\right)=(0,0)$ and decreases quadratically when $q_{1}^{0}$ and $q_{2}^{0}$ increase according to (23) and (19). Its derivatives are also larger for high $\psi$.

Fig. 8b illustrates the influence of the mechanical $Q$ factor on the stability. Once again, a minimum quality factor is required to destabilize the system. Indeed, when decreasing $Q$, the decay rate of the first mode given in Fig. 7b is shifted to a smaller magnitude. Thus, for "small" $Q$ the maximum value of $\sigma(v)$ due to the stabilization of $i_{e, \tilde{u}}$ is negative and no instability can occur. Note that the high numerical $Q$ factor causing instability are in good agreement with the experimental ones [2].

Finally, the last stability map investigated in this section is given in Fig. $8 \mathrm{c}$ and highlights the influence of the time constant ratio $r=\omega R C_{r e f}$ on the nanowire stability. The system is unstable only if the time constant of the mechanical oscillator and electrical $R C$ circuit are of the same order of magnitude. In fact, if this condition is not fulfilled, the energy associated with the destabilizing electrical term $i_{e, \tilde{q}}$ (Appendix A) is no longer supplied to the first mechanical mode. Fig. 8b seems to be the most relevant numerical results to explain the lack of selfoscillations observed when using the field emission configuration with carbon nanotubes since they have an electrical resistance of approximately $10^{5} \Omega$ leading to a time constant ratio $r=4.458 \times 10^{-5}$.

\subsection{Limit cycles}

In this last part, we investigate the nonlinear dynamics of the bi-articulated nanowire in field emission. The purpose is to determine the nanowire equilibrium after initial perturbations of the base state branches given in Fig. 6. This equilibrium is numerically simulated by directly integrated the generalized coordinates $q_{1}(\tau), q_{2}(\tau)$ and $u(\tau)$ from the dimensionless governing

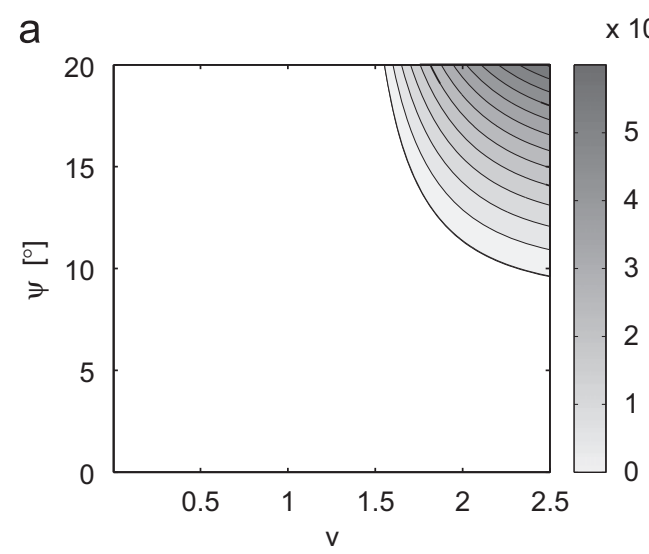

C $\times 10^{-4} \mathrm{~b} \quad \times 10^{4}$

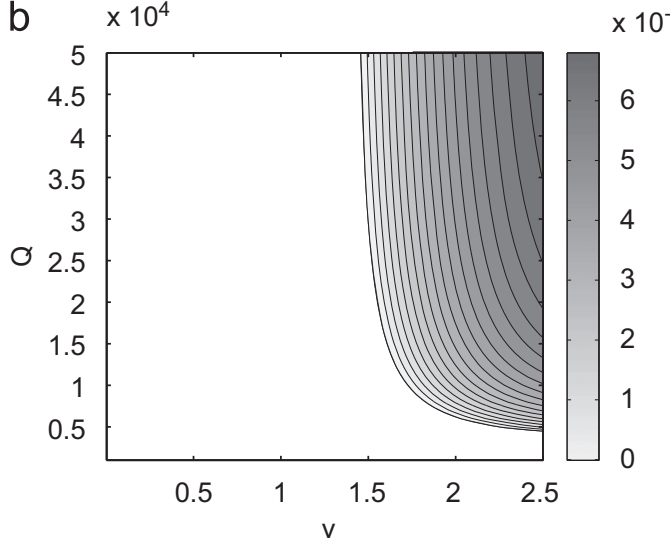

$\times 10^{-4}$

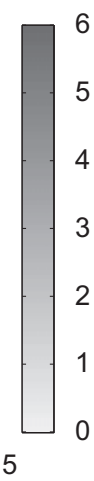

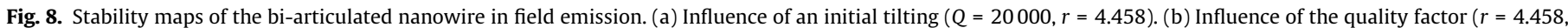
$\left.\psi=20^{\circ}\right)$. (c) Influence of the time constant ratio $\left(\psi=20^{\circ}, Q=20000\right)$. 
Eq. (16). For practical purpose, this fully implicit differential equation is expressed in the form

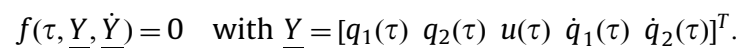

The differential system (28) is solved for an applied $D C$ voltage $v$ and the initial conditions $q_{1}(0), q_{2}(0)$ and $u(0)$ are the fixed points $q_{1}^{0}, q_{2}^{0}$ and $u_{0}$ computed from Eq. (21). The initial perturbations are applied on the initial angular velocities $\phi_{1}(0)=\dot{q}_{1}(0)$ and $\phi_{2}(0)=\dot{q}_{2}(0)$. The electrical quantities $b, c$ and $\mathrm{m}$ are the polynomial regressions of the generalized coordinates $q_{1}$ and $q_{2}$ given in (19) and (20). Since they are continuous functions, the problem (28) is well defined for any $\tau$.

Fig. 9 represents the dynamic response of the bi-articulated nanowire in field emission after damping of the transient for a voltage $v$ slightly superior $(0.1 \%)$ to the instability threshold given in Fig. 7b. The limit cycles plotted in the phase space of the Fig. 9a illustrate the nanowire vibration around its static equilibrium position. The electromechanical model thus seems to account for the experimental self-oscillations observed in [9]. Fig. 9c shows the Power Spectral Density of the steady-state responses $q_{1}(\tau)$ and $q_{2}(\tau)$. At threshold, the mechanical response is harmonic with a frequency $\omega$ given by the linear analysis in Fig. 7a.

Fig. 9b displays as well the self-oscillations of the electric voltage $u(\tau)$ around its static equilibrium. This time, the secondary harmonic of the steady-state response is not negligible beside the fundamental one $\omega$. In agreement with experimental observations, the electrical signal contains a $2 \omega$ component [9]. Indeed, $u(\tau)$ is oscillating with regard to the absolute position $\left(q_{1}, q_{2}\right)$ and performs also two cycles during a nanowire oscillation period. According to the Fowler-Nordheim formula (12b), the FE current $i_{e}(\tau)$ is oscillating at the same frequency as $u(\tau)$ illustrating the possibility of $D C / A C$ conversion in this unforced device.

\section{Concluding remarks}

The study of the basic mechanical phenomena in NEMS and how they can be best controlled by external parameters is of prime importance in view of exploiting their possibilities in devices especially because new effects come into play at the nanoscale that lead to both complications and opportunities. In particular, the possibilities of self-oscillating nanowires in field emission shown by Ayari et al. [9] is an important step toward making NEMS active rather than passive devices but deserves further investigations to understand and also control this physical phenomenon.

This paper gives a low-dimensional model to simulate the nonlinear behaviour of a cantilevered nanowire in field emission. The numerical method was presented with a bi-articulated nanowire but can easily be adapted to another kinematics for obtaining quantitative results rather than qualitative ones. We here highlighted the ins and outs of this electromechanical system resulting from an original coupling between the nanostructure nonlinear motion, its electrostatic environment and electrical contributions coming from the FE current emergence. For a given applied voltage, the linear stability of the static equilibrium results from the interplay of the FE current dependence on the nanowire absolute position and its dependence on the emitter's voltage. This interplay, illustrated by the FowlerNordheim formula, is very sensitive to external parameters and the same is also true for the emergence of oscillations. We showed that a minimum initial angular tilting, a high $Q$ factor and a sufficiently high electrical resistance are required to trigger instability.

For a threshold of applied voltage, the direct integration of the nonlinear electromechanical governing equations simulates the
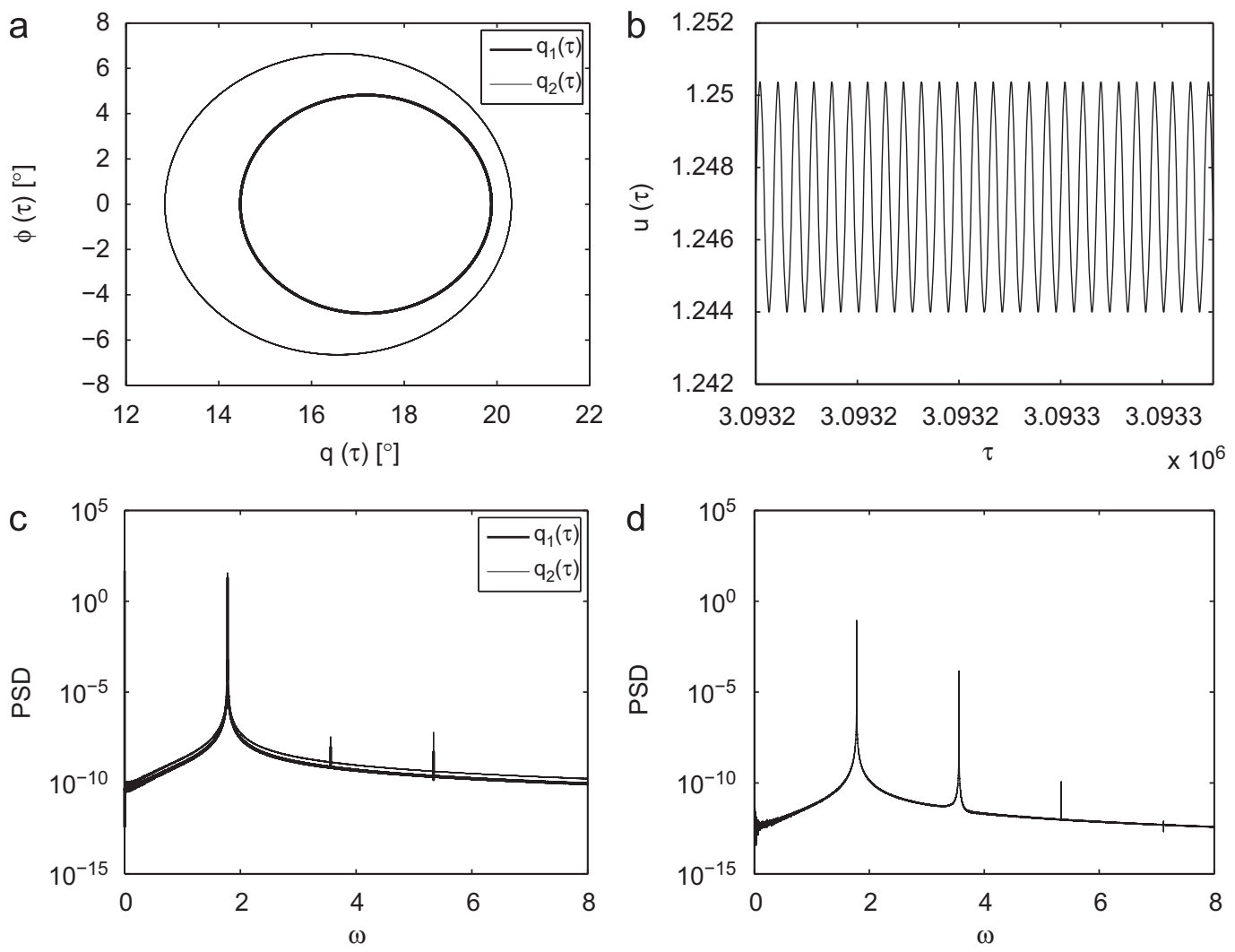

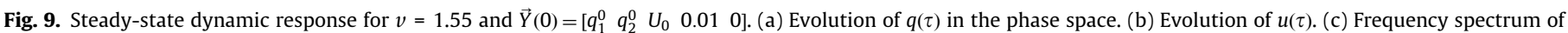
$q(\tau))$. (d) Frequency spectrum of $u(\tau)$. 
limit cycles of the system pointing out the possibility of $D C / A C$ conversion in this electromechanical device. The dimension of the electromechanical system (16) and its strong linearities are sufficient to display complex behaviour of the limit cycles when increasing the control parameter $v$ [21]. Future work would focus more on this original Hopf bifurcation to improve the physical understanding of the self-oscillations.

\section{Appendix A. Simplified model for the field emission instability}

A simpler kinematic model can be used to approximate the FE instability by setting $q(t)=q_{1}(t)=q_{2}(t)$ at any time $t$ (straight nanowire case). Even if this basic model contains a poorer kinematics than its two degrees-of-freedom counterpart (specially not taking in account follower forces), it is sufficient to capture properly the main features of the instability mechanism. Deriving from Eq. (21), the fixed points $\left(q^{0}, u_{0}\right)$ of the straight cantilevered nanowire in field emission satisfy the two degrees-of-freedom electromechanical equations

$v-u_{0}-h b^{2} u_{0}^{2} e^{\left(g / b u_{0}\right)}=0$

$q^{0}-\psi-\mathfrak{m}_{A} u_{0}^{2}=0$

where the dimensionless moment $m_{A}\left(q^{0}\right)$ comes from the external electrostatic forces applied on the straight nanostructure. Fig. 10 displays the evolution of the dimensionless FE current in the $(q, u)$ space given by the Fowler-Nordheim formula $i_{e}=h b^{2} u_{0}^{2} e^{\left(g / b u_{0}\right)}$ where $b\left(q_{0}\right)$ is computed from the electrostatic problem with a straight nanowire. The electromechanical parameters used in Fig. 10 are given in Section 3. The linearized governing equations around $\left(q^{0}\right.$, $u_{0}$ ) derive from (22) and read

$r c \dot{\tilde{u}}+\left(1+i_{e, \tilde{u}}\right) \tilde{u}=-r u_{0} c_{, \tilde{q}} \dot{\tilde{q}}-i_{e, \tilde{q}} \tilde{q}$,

$\ddot{\tilde{q}}+\frac{3}{Q} \dot{\tilde{q}}+\left(3-3 \psi-3 u_{0}^{2} \mathrm{~m}_{A, \tilde{q}}\right) \tilde{q}=6 u_{0} \mathrm{~m}_{A} \tilde{u}$,

where the subscripts ( $)_{, \tilde{q}}$ and ( ) $\tilde{u}_{\tilde{u}}$ respectively represent the derivatives with respect of $\tilde{q}$ and $\tilde{u}$. Finally, by expressing the coupled Eq. (A.2) in the modal basis $Z(\tau)=[\tilde{q}(\tau) \quad \tilde{u}(\tau)]^{T}=$ $\left[\begin{array}{ll}\tilde{q}^{A} & \tilde{u}^{A}\end{array}\right]^{T} e^{s \tau}$, we find for the decay rate of the mechanical prevailing mode $\underline{\varphi}_{1}$ :

$\sigma(v)=-\frac{3}{Q}+\Gamma(v)$

with $\Gamma(v)=\frac{6 r u_{0} \mathrm{~m}_{A}}{r^{2} \omega^{2}+\left(1+i_{e, \tilde{u}}\right)^{2}} \times\left[i_{e, \tilde{q}}-\left(1+i_{e, \tilde{u}}\right) u_{0} c_{, \tilde{q}}\right]$.

Relation (A.3) governs the stability of the straight nanowire static equilibrium against $v$ implicitly through the dependence of the fixed points $\left(q^{0}, u_{0}\right)$ given by (A.1). It can also be qualitatively extended to the decay rate of the first mode of the bi-articulated model given in Fig. $7 \mathrm{~b}$ and serves as a support for the understanding of the stability maps in Fig. 8.

For $v<1$, there is no field emission. The FE current and its partial derivatives $i_{e, \tilde{q}}$ and $i_{e, \tilde{u}}$ are negligible (Fig. 10). The only stabilizing term in relation (A.3) comes from the capacitance dependence on $q$ and the decay rate decreases when $v$ increases. This behaviour is in agreement with the evolution of the decay rate of the first mode given in Fig. $7 \mathrm{~b}$.

For $v>1$, i.e. in field emission configuration, the decay rate (A.3) depends on the competition between the destabilizing term $i_{e, \tilde{q}}$ and the stabilizing terms $i_{e, \tilde{u}}$ and $c_{, \tilde{q}}$. At the beginning of the emission, $i_{e, \tilde{q}}$ and $i_{e, \tilde{u}}$, given by (23), are of the same order of magnitude and the instability can occurred if $c_{, \tilde{q}}$ is not too large as

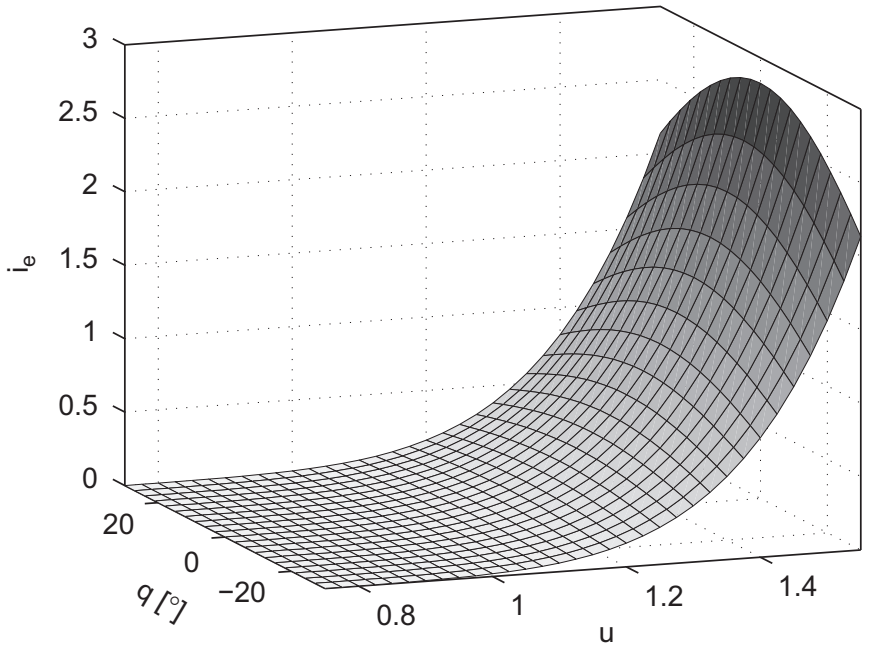

Fig. 10. Evolution of the dimensionless FE current for the straight nanowire.

in Fig. 7b. For higher voltage, since $i_{e, \tilde{u}}$ is increasing faster than $i_{e, \tilde{q}}$, the FE destabilizing effect becomes negligible and $\sigma(v)$ tends to a maximal value before decreasing again due to the stabilizing terms $i_{e, \tilde{u}}$ and $c_{, \tilde{q}}$.

\section{References}

[1] Ekinci KL, Roukes ML. Nanoelectromechanical systems. Review of Scientific Instruments 2005;76:061101.

[2] Perisanu S, Vincent P, Ayari A, Choueib M, Purcell ST, Bechelany M, et al. High $\mathrm{Q}$ factor for mechanical resonances of batch-fabricated SiC nanowires. Applied Physics Letters 2007;90:043113.

[3] Jensen K, Kim K, Zettl A. An atomic-resolution nanomechanical mass sensor. Nature Nanotechnology 2008;3:533-7.

[4] Gammel P, Fischer G, Bouchaud J. RF MEMS and NEMS technology, devices and applications. Bell Labs Technical Journal 2005;10:29-59.

[5] Purcell ST, Vincent P, Journet C, Binh VT. Tuning of nanotubes mechanical resonances by electric field pulling. Physical Review Letters 2002;89:273103.

[6] Bonard J-M, Kind H, Stockli T, Nilsson L-O. Field emission from carbon nanotubes: the first five years. Solid-State Electronics 2001;45:893-914.

[7] Perisanu S, Gouttenoire V, Vincent P, Ayari A, Choueib M, Bechelany M, et al. Mechanical properties of $\mathrm{SiC}$ nanowires determined by scanning electron and field emission microscopies. Physical Review B 2008;77:165434(12).

[8] Perisanu S, Vincent P, Ayari A, Choueib M, Guillot D, Bechelany M, et al. Ultra high sensitive detection of mechanical resonances of nanowires by field emission microscopy. Physica Status Solidi 2007;204:1645-52.

[9] Ayari A, Vincent P, Perisanu S, Choueib M, Gouttenoire V, Bechelany M, et al. Self-oscillations in field emission nanowire mechanical resonators: a nanometric dc-ac conversion. Nano Letters 2007;7:2252-7.

[10] Paidoussis MP. Fluid-structure interactions: slender structures and axial flow, vol. 1. New York: Academic Press; 1998.

[11] de Langre E, Paidoussis MP, Doare O, Modarres-Sadheghi Y. Flutter of long flexible cylinders in axial flow. Journal of Fluid Mechanics 2007:571:371-91.

[12] Gibson RF, Ayorinde EE, Wen Y-F. Vibrations of carbon nanotubes and their composites: a review. Composites Science and Technology 2006;67:1-28.

[13] Vanderlinde J. Classical electromagnetic theory. New York: Wiley; 1993.

[14] Bleaney BI, Bleaney B. Electricity and magnetism, vol. 1. Oxford: Oxford Science Publications; 1991.

[15] Bolotin VV. Dynamic stability of elastic systems. San Fransisco: Holden-day; 1964.

[16] Champneys AR. Homoclinic orbits in the dynamics of articulated pipes conveying fluid. Nonlinearity 1991;4:747-74.

[17] Blevins RD. Formulas for natural frequency and mode shape, vol. 1. New York: Krieger Publishing Company; 1979.

[18] Verpeaux P, Charras T, Millard A. Castem 2000: Une approche moderne du calcul des structures, Calcul des structures et intelligence artificielle, Pluralis, Paris, France, 1988. p. 261-71.

[19] Moin P. Fundamentals of engineering. numerical analysis. Cambridge: Cambridge University Press; 2001.

[20] Langthjem MA, Sugiyama Y. Dynamic stability of columns subjected to follower loads: a survey. Journal of Sound and Vibration 2000;238:809-51.

[21] Manneville P. Instabilities, chaos and turbulence. Imperial College Press; 2004. 